\title{
Estado da Arte em Web Semântica e Web 2.0: Potencialidades e Ten- dências da Nova Geração de Ambientes de Ensino na Internet
}

\author{
Seiji Isotani \\ The Institute of Scientific and Industrial Research \\ Osaka University, Japan \\ isotani@acm.org \\ Riichiro Mizoguchi \\ The Institute of Scientific and Industrial Research \\ Osaka University, Japan \\ miz@ei.sanken.osaka-u.ac.jp
}

\author{
Ig Ibert Bittencourt \\ Universidade Federal do Alagoas, Maceió, AL, Brazil \\ Universidade Federal de Campina Grande, Campina \\ Grande, PB, Brasil \\ ibert@dsc.ufcg.edu.br \\ Evandro Costa \\ Instituto de Computação \\ Universidade Federal do Alagoas, Maceió, AL, Brasil \\ ebc@ fapeal.br
}

\begin{abstract}
Resumo As tecnologias educacionais com base na Web têm obtido excelente resultados nas últimas décadas. Atualmente, diversos cursos no exterior e no Brasil são oferecidos na modalidade "educação a distância” onde a Internet é a plataforma base de comunicação e interação entre alunos e professores. Com os grandes avanços da Internet e a necessidade de oferecer formas mais eficazes de aprendizagem na Web, duas linhas de pesquisa estão em crescente expansão. A primeira delas é a Web Semântica que desenvolve tecnologias que permitem ao computador compartilhar e manipular as informações contidas na Web de forma adequada e inteligente. Com o uso desta tecnologia ambientes de aprendizagem ou agentes de software podem interagir entre si, trocar informações e auxiliar professores e alunos a selecionar, combinar e classificar o conteúdo disponível na Web. E a segunda é a Web 2.0, ou web Social, onde os usuários são beneficiados por diversas ferramentas para compartilhar e construir "conhecimento" de forma simples, interativa e colaborativa. O objetivo deste trabalho é estimular as pesquisas nacionais nestas áreas apresentando o estado da arte sobre o uso da Web Semântica e da Web 2.0 no cenário educacional e a recente interseção destas tecnologias para promover a nova geração de ambientes educacionais para Web 3.0.
\end{abstract}

Palavras-Chave: Web Semântica, Web 2.0, Web Semântica Social, ambientes de ensino.

\begin{abstract}
Web-based educational technologies have been obtaining excellent results in the last decades. Currently, several courses in Brazil and abroad are offered in the modality "distance education" where the Internet is the main platform for communication and interaction between students and teachers. With the great advances of the Internet and the need to offer more effective forms of learning on the Web, two fields of research are in increasing expansion. The first one is the Semantic Web which develops technologies that allows for computers to share and to manipulate the information on the Web in an appropriate and intelligent way. Through this technology learning environments or pedagogical agents can interact among themselves, to exchange information and help teachers and students to select, combine and classify the available content on the Web. And the second one is the Web 2.0 where users can use several tools to share and to build "knowledge" in a simple, interactive and collaborative way. The main objective of this work is to stimulate the research on these fields in Brazil presenting the state of the art on the Semantic Web and Web 2.0 in educational contexts. Furthermore we will also present the recent research directions in which these two fields intersects to promote the new generation of educational environments for the Web 3.0.
\end{abstract}

Keywords: Semantic Web, Web 2.0, Social Semantic Web, learning environments 


\section{Introdução}

Os ambientes virtuais de aprendizagem baseados na Web têm recebido grande atenção por parte da comunidade acadêmica interessada em tópicos relacionados à Internet e a Inteligência Artificial Aplicada à Educação [4]. Atualmente, as principais razões são a crescente evolução das pesquisas em Web Semântica e seu grande potencial para resolver diversos problemas encontrados nos ambientes educacionais baseados na Web atual como, por exemplo, a falta de interoperabilidade entre sistemas e conteúdo, dificuldades para criar e reutilizar a informação, buscas não inteligentes, dentre outros.

Através do uso de técnicas da Web Semântica, a Web tradicional que conecta apenas informação (links entre páginas/mídias), está se transformando na Web que potencialmente conecta "conhecimento", ou seja, as informações conectadas possuem significados que são compreensíveis e compartilháveis por pessoas e computadores [8]. O uso de ontologias e taxonomias para representar conhecimento e anotar a informação contida na Web permitiu o desenvolvimento de novos mecanismos de buscas (buscas semânticas), inferências e análise em banco de dados, além de viabilizar o uso de agentes inteligentes para buscar informação na Web de forma muito mais rápida e efetiva [4]. Contudo, criar ontologias/taxonomias e anotar o conteúdo de forma estruturada é um processo complexo e demorado. Por isso, a Web Semântica ainda encontra dificuldades para ser adotada em cenários menos "acadêmicos".

Em contrapartida, atualmente as ferramentas de colaboração e relação interpessoal na Web são utilizadas em todo mundo [20;32]. No Brasil, o site de relacionamento mais conhecido é o Orkut, enquanto que no exterior o FaceBook e o MySpace são os mais utilizados. Além disso, a criação de blogs, o uso de Wikis, o compartilhamento de fotos, vídeos e lista de favoritos são práticas comuns entre a maioria dos jovens e pessoas que usam Internet. Esta "nova" forma de utilizar a Web é conhecida com Web social ou Web 2.0. A informação contida na Web 2.0 é utilizada e avaliada por outras pessoas que interagem com o conteúdo e seus criadores. Através de tags, pontuações e comentários, existe um envolvimento entre a informação e seus leitores/avaliadores e, dessa forma, apenas as informações mais relevantes acabam prevalecendo [24]. Esta "seleção natural" da informação contida na Web é muito útil quando nos referimos à conteúdos educacionais. Um dos grandes exemplos é a Wikipedia (http://www.wikipedia.org), a maior enciclopédia on-line que possui características singulares como a con- tínua revisão da informação e a inclusão de dados de forma simples, rápida e dinâmica. Contudo, assim como a Web tradicional, a Web 2.0 carece de padrões e linguagem para estruturar e representar a informação (e seu significado) o que dificulta a interoperatibilidade e o reuso do conteúdo (por exemplo, não é possivel compartilhar as informações que um usuário possui no FaceBook para o Orkut e vice-versa).

Para contornar estes problemas, recentemente a comunidade de Web Semântica e Web 2.0 começaram a unir esforços para criar a chamada Web Semântica Social (Social Semantic Web), ou Web 3.0, onde será possível criar sistemas de conhecimento coletivos (Collective Knowledge Systems) na qual as pessoas da comunidade poderão compartilhar a informação como na Web 2.0 e organizar e estruturar o significado destas informações como na Web Semântica [17]. Como resultado, em sistemas educacionais inteligentes, alunos poderão compartilhar informações e recursos que acharem interessantes; agentes inteligentes poderão guiar os estudos buscando e apresentando o melhor conteúdo didático disponível que satisfaça as necessidades dos alunos; grupos de estudos poderão ser formados de forma muito mais eficiente; alunos poderão criar conteúdo de forma ativa e colaborativa; o planejamento instrucional terá suporte inteligente; além de diversos outros benefícios que possivelmente irão emergir com estas novas possibilidades de interação.

No cenário internacional, existe uma forte comunidade realizando pesquisas em Web Semântica aplicada à Educação. Tal comunidade organiza desde 2002 uma série de Workshops (e.g., International Workshop on Applications of Semantic Web Technologies for ELearning) que tem contribuído para o desenvolvimento da área. Contudo, no Brasil a comunidade de Web Semântica ainda é muito pequena. No SBIE2007, iniciou-se uma tentativa de fortalecer as pesquisas nacionais nesta área através do primeiro Workshop de Web Semântica na Educação. Para auxiliar esta iniciativa, o principal objetivo deste trabalho é apresentar o estado da arte relacionado ao desenvolvimento e uso da Web Semântica e da Web 2.0 na Educação. Além disso, será discutida a tendência atual na área de combinar os benefícios destas duas linhas de pesquisa para proporcionar sistemas educacionais inteligentes com grande potencial de difusão e de utilização em situações reais de ensino. Dessa forma, pretende-se estimular leitores e pesquisadores a desenvolverem trabalhos relacionados à Web Semântica Social aumentando a qualidade e a diversidade das pesquisas nacionais nesta área em crescente expansão. 


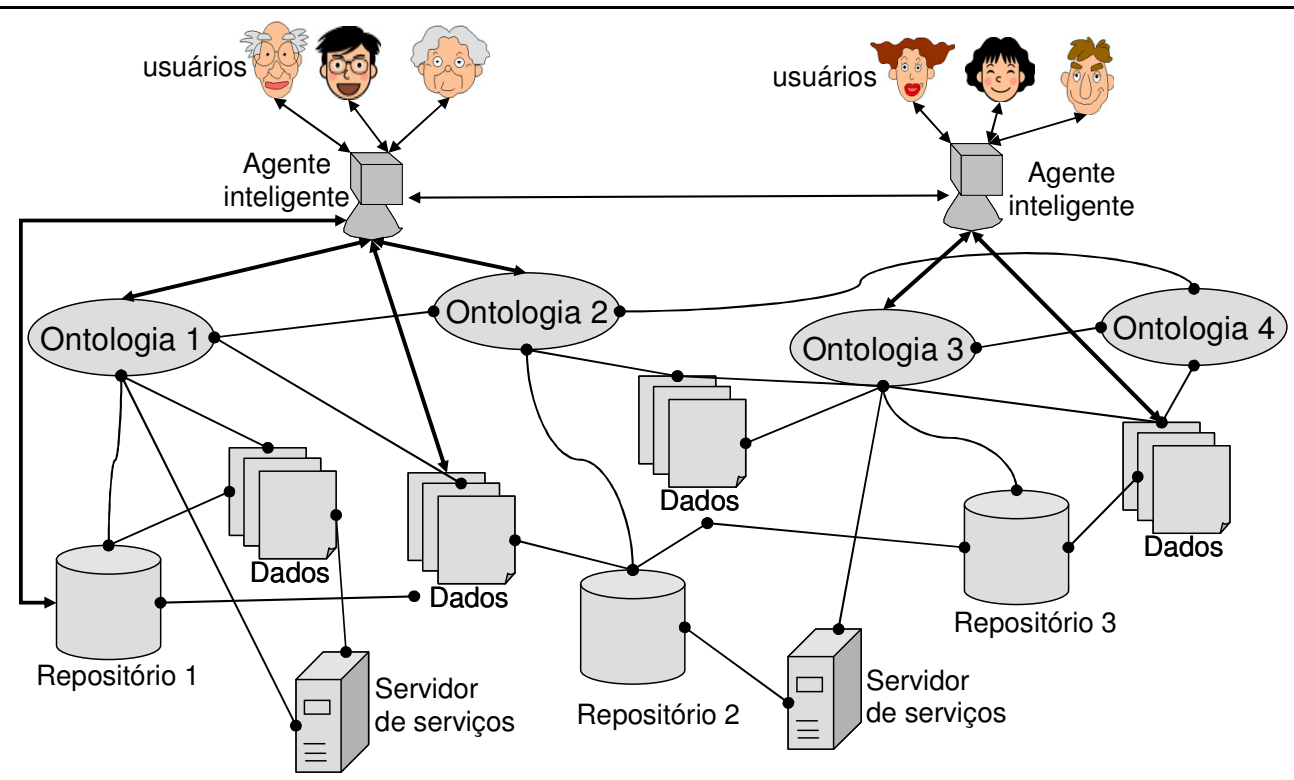

Figura 1. A Visão da Web Semântica onde o conteúdo está distribuído na Web e anotado utilizando diferentes ontologias permitindo que agentes inteligentes possam compreender e utilizar a informação (as linhas e setas representam algumas das possíveis conexões/interações entre objetos).

\section{A Web Semântica e sua Aplicação na Educação}

A informação na Web é tipicamente representada em linguagem natural permitindo que ela seja compreendida por pessoas. Entretanto para prover informação de forma que computadores ou agentes de software possam compreendê-la (e extrair seu significado) é necessário representá-la formalmente e de maneira sistemática. Dessa forma, é possível atribuir semântica aos dados disponíveis na Web. A Web Semântica foi o nome utilizado para introduzir a nova geração de tecnologias que tem como objetivo representar a informação de uma maneira na qual computadores sejam capazes de interpretá-la. Além disso, através desta representação as pesquisas em Web Semântica propõem tecnologias para automação, integração e reuso da informação, mesmo considerando diferentes plataformas de desenvolvimento, sistemas operacionais, protocolos de rede, e outras variações de tecnologia [8]. Atualmente este é um dos principais tópicos de pesquisa das comunidades de Inteligência Artificial e de Internet [33].

Segundo Mizoguchi et al. [24], as ontologias são consideradas a base da Web Semântica, oferecendo uma linguagem expressiva e formal para gerar informação que possa ser interpretada por computadores. Estas ontologias (normalmente descritas em OWL [28]) podem ser combinadas, compartilhadas, modificadas e utilizadas para anotar "semanticamente" diferentes tipos de recursos como, páginas Web, documentos, mídias (digitais ou não), além de outros recursos [20]. Dessa forma, as onto- logias oferecem a possibilidade de incluir significado na informação descrita na Web permitindo que os computadores "raciocinem" (realizem inferências) sobre os dados disponíveis na Web de forma mais "inteligente". Segundo [8], o uso de ontologias e o desenvolvimento de serviços Web para processar a informação disponível na Internet está transformando a Web da informação (Web tradicional) na Web do conhecimento. Como mostra a Figura 1, a visão da Web Semântica é criar a Web do conhecimento na qual a informação está distribuída em diferentes repositórios e anotada utilizando ontologias interconectadas. Através destas ontologias, agentes de software inteligentes podem acessar, compartilhar e trocar informações de maneira eficiente, facilitando o desenvolvimento de serviços que agreguem dados de diferentes localidades. Essa mudança de paradigma na Web permite que computadores e pessoas trabalhem cooperativamente de maneira muito mais eficiente [1].

No contexto educacional, a Web Semântica e ontologias têm sido utilizadas para resolver diversos problemas encontrados nos atuais ambientes educacionais baseados na Web. Alguns problemas como a dificuldade no compartilhamento e reuso de material didático, a busca por conteúdo (ex. objetos de aprendizagem) e o gerenciamento de repositórios de objetos de aprendizagem, podem ser resolvidos melhor utilizando e adaptando os conceitos da Web Semântica como os apresentados na Figura 1. De fato, alguns resultados apresentados pela comunidade de Inteligência Artificial aplicada à Educação mostram que os ambientes de ensino baseados na Web Semântica podem proporcionar resultados sensívelmente positivos se comparados aos ambientes de ensino baseados na Web 
atual $[8 ; 10 ; 18]$. Muitos destes resultados estão disponíveis gratuitamente no portal O4E - Ontologies for Education (http://o4e.iiscs.wssu.edu/xwiki).

Por exemplo, no trabalho de Mizoguchi et al. [24], propôs um conjunto de ontologias para representar o planejmento instrucional baseado em diferentes teorias instrucionais e de aprendizagem. Através do uso destas ontologias, os autores desenvolveram um sistema inteligente de suporte ao professor que o auxilia durante o planejamento de atividades de ensino. Essas ontologias também podem ser utilizadas em sistemas de educação a distância permitindo que todas as atividades do curso tenham suporte pedagógico e se adaptem conforme o desenvolvimento do aluno. Além disso, os professores podem receber recomendações do ambiente de ensino e utilizar diferentes abordagens pedagógicas para atingir seus objetivos. Esse tipo de suporte pedagógico, semiautomático, que analisa as diversas variáves do ambiente (estado do aluno, estilos de aprendizagem, preferências do professor, e etc) e, se adapta de forma dinâmica e inteligente é de grande importância na Educação a distância onde professores e alunos não estão "conectados" fisicamente.

Em outro trabalho, Dicheva e Dichev [13] apresentam o TM4L - Topics Maps for eLearning, uma biblioteca de conteúdo/curso digital baseado na Web Semântica que auxilia professores e alunos a procurar, usar (reusar) e compartilhar material didático através do uso de mapas conceituais. O TM4L oferece ferramentas de busca poderosas, e intuitivas permitindo que os usuários (alunos e professores) encontrem e utilizem recursos educacionais de maneira mais efetiva. Além disso, este programa também oferece ferramentas de autoria para criação e modificação de conteúdo.

Atualmente, diversas pesquisas em sistemas adaptativos hipermídia utilizados na educação estão direcionadas ao desenvolvimento de arcabouços (frameworks) e métodos que viabilizem a interoperabilidade entre diferentes sistemas através dos recursos da Web Semântica. Por exemplo, os trabalhos de De Bra et al. [10] e Motta et al. [26] propõe a modularização dos diversos compenentes pertencentes à arquitetura dos sistemas hipermídia para permitir que serviços semânticos e ontologias possam ser incorporadas e utilizadas adequadamente.

Além dos exemplos citados, formas inteligentes de oferecer feedback, modelos de aluno mais flexíveis e apresentação dinâmica de conteúdo educacional são tópicos de grande interesse da comunidade de Web Semântica na Educação [5;11;31].

Em resumo, pode-se dizer que a Web Semântica propõe tecnologias que dão mais "poder" aos computadores através da criação de padrões, protocolos e linguagens formais que facilitam o processamento da informação na Web de forma automática e inteligente. Nas próximas sub-seções serão apresentados algumas das tecnologias da Web semântica que vêm auxiliando o desenvolvimento de ambientes educacionais, principalmente durante a arquitetura e padronização destes sistemas.

\subsection{Arquitetura}

Atualmente, os sistemas de aprendizagem na Web possuem pouca ou nenhuma interoperabilidade entre si. Isso significa que praticamente toda a informação disponível num determinado sistema não pode ser compartilhada com outros sistemas. Por exemplo, as interações e os estilos de aprendizagem de um aluno, não podem ser compartilhadas, pois a forma de representar o modelo do aluno em cada sistema é diferente. Outra dificuldade é o compartilhamento de material educacional, cursos ou preferências pedagógicas. Apesar de alguns sistemas adotarem padronizações de linguagem para formatar cursos (eg. IMS-LD) e seus materiais, conhecidos como objetos de aprendizagem (eg. LOM), a arquitetura dos atuais sistemas não permite um melhor compartilhamento e interoperabilidade da informação. Por exemplo, mesmo que um curso esteja representado em IMS-LD e os objetos de aprendizagem estejam em LOM, informações mais detalhadas sobre o uso dos cursos e/ou dos materiais não podem ser compartilhadas.

Para resolver este problema a Web semântica oferece modelos de referência que podem ser utilizados por qualquer sistema como base para o desenvolvimento de aplicativos educacionais que permitem grande interoperabilidade, para a troca e o compartilhamento de informação, além de permitir que um sistema seja decomposto e construído em módulos. Assim, um novo sistema pode ser construído a partir de módulos de outros sistemas, ou utilizar parte de outros sistemas para compor algumas de suas funcionalidades. Nestes modelos de referência são descritos princípios de desenvolvimento, papéis para usuários, fluxo da informação entre módulos e usuários, além de diversas outras informações.

Dois modelos de referência utilizados para definir a arquitetura dos sistemas baseados na Web semântica podem ser encontrados nos trabalhos de [4] e [8]. Apesar de cada modelo possuir algumas características particulares, ambos compartilham os conceitos fundamentais para desenvolver sistemas educacionais com tecnologia semântica. A seguir, serão descritos estes principais conceitos:

\section{- Papel do usuário.}

Em sistemas educacionais baseados em Web semântica é necessario distinguir os papéis disponíveis para os usuários do sistema. Assim, é possível restringir 
acesso a algumas partes do conteúdo, armazenar a informação da interação entre usuário e sistema de maneira coerente, facilitar a navegação no ambiente, auxiliar na criação, edição e organização do conteúdo, além de diversas outras facilidades. Os papéis mais utilizados são: professor (responsável por definir as estratégias pedagógicas e monitorar/auxiliar os alunos), aluno, autor (responsável por criar/armazenar e organizar o material educacional), desenvolvedor (responsável por desenvolver novas funcionalidades no sistema) e comunidade (este papel representa um grupo de alunos que desenvolve atividades colaborativas).

\section{- Recursos Educacionais e Interface}

Recursos educacionais são as ferramentas disponíveis no ambiente para criar, armazenar e organizar o conteúdo educacional. Alguns exemplos de recursos educacionais são: os objetos de aprendizagem (textos, exercícios, cursos, atividades, etc), serviços educacionais semânticos (utilizados para composição de cursos e outras tarefas internas do sistema), ontologias educacionais, além de outros. Estes recursos estão disponíveis ao usuário (com um papel específico) através da interface do sistema. Para o usuário, os processos internos que ocorrem no sistema não são apresentados na interface. Por exemplo, um objeto de aprendizagem que está em outro ambiente pode ter sido localizado por um agente pedagógico e adaptado para se adequar às necessidades do usuário. Todavia, na interface do sistema, o usuário terá a impressão que o objeto de aprendizagem se encontra armazenado localmente e tem as características desejadas por ele. Assim, a interface de sistemas baseados na Web semântica deve ser adaptável o suficiente para considerar o papel e as necessidades do usuário (ex. para usuários autores apenas ferramentas de autoria estarão visíveis e disponíveis), seu comportamento dentro do sistema, e os serviços disponíveis internamente que possibilitam a composição e o oferecimento de diversas outras funcionalidades que facilitam a interação entre o usuário e o ambiente educacional.

\section{- Suporte Estrutural}

Como discutido anteriormente, uma das grandes vantagens de se utilizar tecnologias da Web semântica é a possibilidade de criar novos mecanismos que permitam um melhor uso da informação pelos computadores, facilitando a interoperabilidade entre sistemas computacionais. Para oferecer tais mecanismos é necessário um suporte estrutural, tal como apresentado na Figura 1. As ontologias são essenciais para garantir (pelo menos parcialmente) a interoperabilidade e o uso das informações contidas nos sistemas educacionais. São elas as responsáveis por representar a informação formalmente, permitindo que computadores re- alizem inferências para produzir um comportamento mais "inteligente". Os agentes pedagógicos podem utilizar as ontologias para ajudar os usuários em diversas tarefas. Por exemplo, um agente pode auxiliar/guiar um aluno com dificuldade durante a resolução de uma atividade. Um outro agente, pode ajudar o professor a localizar objetos de aprendizagem para compor suas aulas. Assim, os agentes são essenciais para interagir com os usuários garantindo um melhor processo de aprendizagem pelos alunos e facilitando o planejamento de atividades educacionais pelos professores. Os recursos educacionais ficam armazenados em diferentes repositórios e podem ser acessados por usuários e agentes de diferentes sistemas. Portanto, é essencial que os recursos educacionais sejam armazenados utilizando alguma linguagem padronizada, pois mesmo que um repositório utilize uma padronização diferente dos demais, através do uso de ontologias, é possível fazer o mapeamento das informações contidas nos diversos repositórios e extrair o conteúdo necessário. Finalmente, devido a distribuição dos dados na Web, nem sempre as informações estão disponíveis para qualquer ambiente. De fato, existem servidores especiais que oferecem serviços que podem ser "acoplados" ao ambiente de forma transparente. Assim, é possível acessar dados de forma segura e também descrobrir, compor e invocar serviços dinamicamente para prover maiores funcionalidades ao sistema. Tais serviços também são especificados semanticamente, através da utilização de ontologias, permitindo assim que a descoberta, composição e invocação dos mesmos ocorram de forma automática pelos agentes de software.

\subsection{Padronização}

Como abordado anteriormente, a padronização dos recursos disponibilizados na Web é de fundamental importância para descrever, desenvolver, acessar, anotar, combinar e qualificar tais recursos. De fato, há padrões definidos tanto para a especificação de ontologias e serviços para a Web Semântica, quanto para os recursos educacionais. Dentre os padrões disponibilizados atualmente, destacam-se:

\section{- Ontologias na Web Semântica}

A linguagem OWL (Ontology Web Language) é o padrão utilizado pela comunidade de Web Semântica para descrever ontologias. Ela permite expressividade de alto nível e inferência implícita. Além disso, esta linguagem possui 3 dialetos diferentes [28], sendo eles a) OWL-Lite: possui pouca expressividade, sendo utilizada mais para classificação herárquica e através de simples restrições como cardinalidade e interseção de classes e restrições, b) OWL-DL: garante mais ex- 
pressividade que OWL-Lite, permitindo construções mais complexos baseadas na lógica de descrição ${ }^{1}$. Além disso, OWL-DL possui propriedades que podem ser definidas com as características de inversa, funcional inversa, simétrica e transitiva. Desta forma, esta linguagem faz inferências em busca de novos conhecimentos e garante decidibilidade [2] e c) OWL-Full: esta linguagem possui mais expressividade que as anteriores permitindo a utilização de todos os recursos disponibilizados na OWL-DL e também dos construtores RDF (Resource Description Framework). Entretanto, esta linguagem não garante decidibilidade. Com isso, deve-se avaliar as necessidades de cada situação na qual se pretende utilizar ontologias e, com isso, decidir qual dos dialetos utilizar;

\section{- Outros padrões da Web Semântica}

Além da linguagem OWL, há diversos outros padrões na Web Semântica que são utilizados, como para regras, linguagem de consulta, serviços Web semânticos, entre outros. Em 2004, a linguagem para descrição de regras na Web Semântica, SWRL (Semantic Web Rule Language), foi submetida para recomendação do W3C. Já a linguagem de consulta, SPARQL virou recomendação do W3C em 2008. Para a descrição de serviços Web semânticos [23], a linguagem OWL-S (OWL for Services) foi proposta, fazendo uma extensão do wsdl, descrito em XML para a descrição através de OWL [22].

\section{- Padrões Educacionais}

A utilização dos padrões para educação é motivada por diversas razões como por exemplo, potencializar o reuso e compartilhamento dos recursos e permitir a interoperabilidade entre diferentes ambientes educacionais. Além disso, tais padrões possuem propósitos diferentes como padrões de metadados, de informações dos estudantes, comunicação, qualidade, entre outros. Alguns padrões que são destacados são [9]: a) LOM (Learning Object Metadata): a comunidade de informática e educação tem utilizado o termo "Objetos de Aprendizagem" para descrever os recursos educacionais. LOM equivale à uma iniciativa da IEEE LTSC, onde propôs o Padrão de matadados para objetos de aprendizagem. Este padrão é dividido em nove categorias, descrevendo informações gerais sobre os objetos de aprendizagem, ciclo de vida, informações sobre os metadados, requisitos técnicos, características pedagógicas, direitos autorais, relação com outros padrões, anotação e classificação. b) IMS-LD (Learning

\footnotetext{
${ }^{1}$ A versão atual da OWL-DL $\operatorname{SHOIN}{ }^{(\mathrm{D})}$, Entretanto, a versão 1.1. da OWL foi submetida para recomendação pelo W3C em 2006 e possui expressividade $S H O I Q^{(\mathrm{D})}$. Frisa-se ainda que em 2008 um rascunho da
} linguagem OWL 2.0 foi submetido ao W3C.
Design): este padrão é utilizado para especificar o projeto instrucional das unidades de aprendizagem, tendo sido proposto pelo IMS Global Learning Consortium. Tal padrão possui diversas abordagens pedagógicas e permite a integração das mesmas em uma unidade de aprendizagem. Além do IMS-LD, este consórcio desenvolveu diversos padrões, como IMSQTI (testes e questões), IMS-CP (empacotamento de conteúdo), IMS-LIP (pacote de informação de estudantes), dentre outros.

É importante frisar que os objetos de aprendizagem são armazenados em repositórios (LOR - Learning Object Repositories) [15]. Desta forma, os ambientes educacionais podem fazer uso dos diversos objetos de aprendizagem.

Além dos padrões educacionais proposto pelo IMS Global Learning Consortium, há também os padrões proposto pela ADL (Advanced Distributed Learning Initiative - http://www.adlnet.gov/), chamados de SCORM (Shareable Content Object Reference Model). SCORM equivale a um conjunto de padrões educacionais com o objetivo de prover estruturas que possam ser reutilizadas por diversos ambientes educacionais Web. Desta forma, O SCORM é dividido em três partes, sendo eles o i) Modelo de Agregação de conteúdos onde os metadados estão definidos e os objetos de aprendizagem podem ser estruturados e empacotados; ii) Ambientes de Execução para garantir que tais objetos de aprendizagem disponibilizados no SCORM possam ser visualizados pelos ambientes educacionais (principalmente os conhecidos LMS - Learning Management Systems) e iii) Sequênciamento e Navegação, onde pode-se definir regras de sequênciamento para descrever quandos determinado objeto de aprendizagem será mostrado. Com isso, o SCORM busca garantir a padronização, reusabilidade, flexibilidade e interoperabilidade entre ambientes educacionais.

\section{- Padrões Educacionais Semânticos}

Além dos padrões para a Web Semântica e para educação, outros padrões foram propostos através da extensão dos padrões educacionais e sua especificação com linguagens provenientes da Web Semântica. Dentre os padrões educacionais sob a perspectiva da web semântica, citam-se LOM RDF, TANGRAM (ontologia do modelo do usuário baseada nos padrões IMSLIP e IEEE PAPI Learner), ontologia para IMS LD, dentre outros.

Apesar dos grandes benefícios apresentados nesta seção, para o usuário final (internauta, aluno ou professor), a Web Semântica ainda é muito complexa para ser utilizada em ambientes educacionais. Isso ocorre porque a Web Semântica foi concebida para facilitar o consumo da 
informação pelos computadores e, assim, grande parte dos esforços estão relacionados ao suporte estrutural do sistema. Portanto, as aplicações (protótipos) desenvolvidas até o momento, normalmente, não dispõem de uma interface amigável que proporcione maior liberdade ao usuário final. Para suprir esssa deficiência uma "nova" extensão da Web atual, conhecida como Web 2.0, está recebendo grande atenção, tanto dos usuários finais quanto dos pesquisadores.

\section{A Web 2.0 e suas Aplicações na E- ducação}

Enquanto a Web Semântica concede mais poder aos computadores, permitindo que estes possam compreender a informação disponível na Internet, a Web 2.0 previlegia o poder aos usuários. Segundo Murugesan [27], a Web 2.0 transforma o antigo modelo da Web tradicional, onde os usuários são simples consumidores passivos da informação, em uma plataforma social para trocas de experiências através da colaboração. Nesta nova era da Web, os usuários podem se encontrar, colaborar e interagir para criar e compartilhar conhecimento.

Para Mikroyannidis [24], a Web 2.0 pode ser descrita como a Web que dá liberdade aos usuários através das tecnologias para colaboração entre pessoas e da descentralização dos meios produtores de informação. Ou seja, na Web 2.0 qualquer usuário possui as ferramentas e a liberdade necessária para se tornar produtor de informação/conteúdo. Além disso, a informação é criada de forma coletiva e não individual. Através de sites de relacionamento, ferramentas de comunicação síncrona e assíncrona, blogs, Wikis, além de outras formas de colaboração, os usuários podem interagir uns com os outros criando a chamada inteligência acumulada ${ }^{1}$ [17].

A criação e avaliação do conteúdo por pessoas de uma mesma comunidade criam mecanismos sociais que impedem, ou pelo menos dificultam, que informações de baixa qualidade tenham grande impacto dentro da comunidade (ou mesmo fora dela). Esse mecanismo social é um dos conceitos chave da inteligência acumulada e também para o atual sucesso da Web 2.0 [27]. No contexto educacional, a interação contínua entre aprendiz-aprendiz e aprendiz-conteúdo, e a possibilidade de argumentar e compreender diferentes pontes de vista é um dos melhores cenários para uma aprendizagem mais rica e duradoura [32]. Assim, devido ao grande potencial benéfico da Web 2.0 em criar tais cenários educacionais, que abrem

\footnotetext{
${ }^{1}$ Do Inglês collected intelligence. A tradução ao pé da letra seria inteligência reunida/recolhida/coletada. Contudo, o significado do termo é a acumulação do conhecimento através da interação entre pessoas.
}

novas perspectivas pedagógicas, muitos pesquisadores na área de informática na Educação estão propondo e avaliando diversas formas de se trabalhar com essas ferramentas para que o aprendizado seja realizado de forma mais interativa, amigável e com resultados mais efetivos se comparado aos sistemas de educação on-line atuais [14;32].

Nas próximas sub-seções serão apresentados algumas das ferramentas mais utilizadas por usuários da Web 2.0 para facilitar o processo de ensino-aprendizagem na Internet.

\subsection{Wikis}

Uma das ferramentas mais utilizadas atualmente para a realização da inteligência acumulada é o Wiki. De forma resumida, pode-se dizer que um Wiki é uma página Web que pode ser lida e editada por qualquer usuário (devidamenta autorizado) que possua um navegador de Internet (ex. Internet Explorer ou Mozilla) e sem a necessidade de nenhum conhecimento tecnológico avançado.

O primeiro Wiki, chamada WikiWikiWeb, foi desenvolvido em 1994 e oficialmente incluído na Internet em 1995 por Ward Cunnihgham com a promessa de criar um espaço na Web onde os usuários poderiam produzir conteúdo de forma rápida e colaborativa [21].

O grande diferencial de uma simples página na Internet e um Wiki é a possibilidade de criar conteúdo de forma colaborativa, armazenando o histórico de todas as mudanças realizadas na página desde sua primeira publicação. Assim, uma versão antiga da página pode ser comparada com uma versão mais recente, permitindo que os usuários identifiquem o local exato onde ocorreram alterações. Além disso, utilizando um Wiki é possível retornar a uma versão anterior da página com um simples toque de botão.

Devido a simplicidade de uso e a grande versatilidade dos Wikis, atualmente esta ferramentas tem chamado a atenção de diversos professores e educadores. Através dos Wikis é possível incentivar alunos, professores e até mesmo pais de alunos a trabalharem em conjunto em prol da educação. Com isso, alunos podem usar um Wiki para realizar trabalhos em grupo, compartilhar informação com outros alunos, mostrar seu próprio ponto de vista, além de permitir que outras pessoas comentem ou ofereçam ajuda para que a informação compartilhada seja apresentada da melhor maneira possível. Já os professores têm a possibilidade de utilizar um Wiki para criar a estrutura do currículo de forma colaborativa, compartilhar problemas interessantes com seus alunos e incentivar a participação ativa de todos em classe, motivando discussões, troca de idéias e a construção do conhecimento através da exploração do conteúdo e a interação entre 
alunos. Além disso, com o uso dos Wikis, pais de alunos podem mais facilmente interagir com a escola e professores, propondo modificações no currículo escolar, e também verificando os trabalhos escolares realizados pelos alunos e, assim, imergindo de forma mais eficaz no processo de ensino-aprendizagem de seus filhos.

Um dos Wikis mais famosos e utilizados atualmente para compartilhar informação e promover a educação é a Wikipedia, uma enciclopédia on-line construída utilizando a tecnologia Wiki. Nesta enciclopédia on-line, pessoas ao redor do mundo escrevem voluntariamente e, colaborativamente, sobre praticamente qualquer assunto. Apesar de alguns problemas de vandalismo (ex. artigos criados para prejudicar terceiros) e de artigos com informação errônea, a Wikipedia tem se tornado referência na procura por informação/conhecimento. De acordo com [6], o uso dos Wikis e da Wikipedia oferece um novo paradigma de colaboração e publicação de material na Web.

Porém, apenas utilizar o Wiki como ferramenta de ensino e não modificar a forma de ensinar, não produz resultados efetivos. Com a introdução das novas tecnologias, o professor deve livrar-se do estigma de detentor do conhecimento e se transformar em um "assistente" que oferece dicas e estímulos para que os alunos aprendam de forma colaborativa, onde o conhecimento é construído por todos. Nesta abordagem de ensino, o professor será o "parceiro" dos alunos, liderando e propondo atividades que visem a exploração e a descoberta num ambiente social que favorece a criatividade, a interação entre alunos e a discussão sobre o assunto abordado [19].

\subsection{Sites para Compartilhamento de Fotos, Vídeos e Materiais Educacionais}

Outro meio popular de contribuição na era da Web 2.0 está relacionado aos sites de compartilhamento de fotos e vídeos. Em sites como YouTube e Veoh, para compartilhamento de vídeos, e Flickr e Picasa, para compartilhamento de fotos, usuários da Internet podem compartilhar informações úteis que podem ter grande impacto educacional. Por exemplo, é possível disponibilizar na Web tutoriais e tele-aulas, como as do Telecurso 2000 (http://www.telecurso2000.org.br/), ou fotos interessantes que podem servir para ilustrar algum conceito (ex. foto de uma célula que pode ser utilizada durante uma aula de ciências). Dessa forma, qualquer pessoa com acesso a Internet pode se beneficiar destes recursos tendo a sua disponibilidade diferentes conteúdos educacionais, que muitas vezes se complementam e facilitam a compreensão da matéria. Além disso, os próprios usuários podem criar conteúdos multimídias (vídeos, fotos, sons, etc) e disponibilizá-los para a comunidade. Ao receber o conteúdo, a comunidade pode avaliá-lo, fazer comentários (tanto para o criador como para outros usuários), ou pro- por formas de melhorar o conteúdo (ex. um vídeo baseado em um outro já disponível). Ou seja, tanto os consumidores da informação quanto os produtores estão continuamente avaliando e melhorando o conteúdo disponível.

No contexto educacional, os sites de compartilhamento de material conhecidos como opencourseware têm adquirido grande popularidade nas universidade de todo o mundo. Estes sites oferecem de forma gratuita materiais de alta qualidade que são utilizados em seus cursos e podem ser reutilizados a qualquer momento, por qualquer instituição, organização ou indivíduo. Universidades em todo o mundo têm oferecido de forma constante materiais como notas de aula, provas, vídeos de aulas, além de diversos outros recursos utilizados em seus cursos. Alguns exemplos de opencourseware disponíveis na Internet podem ser encontrados nos sites do Instituto de Tecnologia de Massachusetts (MIT - http://ocw.mit.edu/), da Universidade da Califórnia em Irvine (http://ocw.uci.edu/), da Universidade de Osaka (http://ocw.osaka-u.ac.jp/), além de diversas outras universidades. No Brasil, a Universidade de Campinas (Unicamp - http://www.ensinoaberto.unicamp.br/) foi uma das pioneiras em oferecer conteúdo aberto gratuito de seus cursos para usuários da Internet.

Entretanto, com a grande quantidade de material disponível gratuitamente na Web surge novamente o problema de identificar o melhor conteúdo ou fazer buscas por conteúdo de forma adequada. Para contornar este problema, a Web 2.0 oferece aos usuários ferramentas para descrever e anotar o material dispobilizado na Web utilizando rótulos (tags/ folksonomies) que servem como palavras-chave na busca por conteúdo. Assim, outros usuários com interesses similares podem se beneficiar destes rótulos, pois ao fazer uma busca com algumas destas palavras-chave os sistemas podem apresentar de maneira mais rápida e com mais precisão os resultados mais relevantes [17]. Este mecanismo pressupõe que pessoas com interesses semelhantes utilizem os mesmos rótulos, ou rótulos similares para identificar o conteúdo na Web. Embora nem sempre isso ocorra, este mecanismo tem obtido excelentes resultados. Segundo [3], este fenômeno é conhecido como a sabedoria das massas (the wisdom of crowds). A Figura 2 mostra uma das representações mais utilizadas para apresentar a sabedoria das massas com os rótulos mais utilizados para anotar um conjunto de fotos compartilhadas pelo site Flickr.

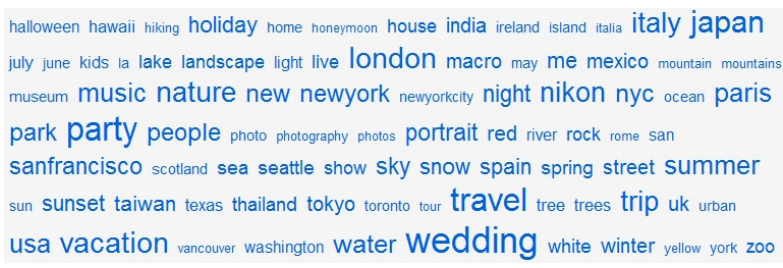


Figura 2. Um exemplo do uso da sabedoria das massas mostrando os rótulos mais utilizados para anotar um determinado conjunto de fotos no site Flickr. Quanto maior o tamanho das letras, maior é a quantidade de pessoas que utilizam estes rótulos.

Através destes materiais disponíveis gratuitamente na Web, é possível criar aulas muito mais interessantes, participativas e interativas. Professores podem utilizar parte do conteúdo disponível para compor notas de aulas, mostrar em sala de aula vídeos que ilustrem o conceito trabalhado e, além disso, propor a leitura de material complementar ou a visualização de vídeos relacionados que podem ser gratuitamente obtidos na Internet. Para os alunos, a possibilidade de adquirir conteúdo de alta qualidade de forma simples, a qualquer hora e em qualquer lugar, incentiva o comportamento investigativo e motiva o aluno a procurar e até mesmo criar conteúdo como vídeos, fotos, wikis e blogs relacionados com o assunto desenvolvido em classe [29].

\subsection{Sites de Relacionamento}

A última ferramenta que será apresentada e a que tem maior popularidade na Web 2.0 é conhecida como site de relacionamento. Estes sites, como o Facebook e o Orkut, permitem que os usuários mantenham contato com seus amigos e familiares, além de viabilizar o compartilhamento de diferentes tipos de conteúdo e a criação de aplicativos (por qualquer usuário) que podem ser inseridos diretamente na página principal. Outro atrativo destes sites é a possiblidade de se criar grupos (conhecidos como $\mathrm{co}$ munidades) com pessoas que possuem interesses em comum. Dessa forma, os sites de relacionamento se tornaram essenciais para qualquer pessoa com acesso a Internet, pois mesmo na vida cotidiana (fora da Internet), seja na escola, na universidade ou no trabalho, as pessoas após interagir na forma presencial, continuam a interagir de modo virtual inserindo e compartilhando fotos e videos, fazendo comentários, propondo novas atividades (presenciais ou não) para o dia seguinte, além de outras formas de colaboração que são utilizadas no meio On-line (chats, foruns, e etc).

Apesar de não serem desenvolvidos com propósitos educacionais, existem atualmente diversos aplicativos voltados para educação que podem ser "anexados" à página principal. A maioria deles foi criado pelos próprios usuários que, muitas vezes, desenvolvem estes pequenos programas para auxiliar em seu próprio aprendizado. Contudo, devido a liberdade que os sites de relacionamento oferecem, esses aplicativos acabam sendo utilizados por milhares de usuários que se interessam sobre o mesmo assunto. Dessa forma, cria-se um ambiente muito mais participativo e motivador onde os próprios usuários podem contribuir para a comunidade.
Um exemplo de um aplicativo para o Facebook com foco educacional é o Kanji box apresentado na Figura 3. Este progarma foi desenvolvido para o ajudar o usuário a ler Japonês. Através de exercícios realizados (leitura de caracteres, tradução de uma palavra, etc), na parte superior direita do aplicativo mostram-se barras que indicam o "nível" de aprendizagem do usuário (quantos caracteres chineses o usuário já aprendeu, quantos ainda faltam, etc). Este nível de aprendizagem fica visível a todos os membros da comunidade para que seja possível criar um ambiente mais colaborativo onde uma pessoa possa verificar quanto conhecimento já adquiriu em comparação aos demais. E assim, permitindo que usuários do mesmo nível compartilhem dúvidas e troquem experiências e, usuários mais avançados, ofereçam suporte e sugestões que podem ser úteis para completar/resolver os exercícios propostos e subir de nível. Além disso, os usuários mais ativos e com maior nível de aprendizagem são destacados no centro da página principal. Este mecanismo, quando utilizado de forma correta, motiva os usuários a continuar entrando no site, fazendo exercícios e estudando para obter uma maior pontuação/nível de aprendizagem para aparecer na página principal.

Dessa forma, pode-se perceber que o uso das ferramentas da Web 2.0 pode trazer diversos benefícios adicionais ao ensino tradicional, principalmente por permitir novas práticas pedagógicas e formas de aprendizagem mais ativas e interativas na qual o próprio aluno pode ser o responsável por criar conteúdo e ferramentas que facilitem/auxiliem o aprendizado. No trabalho de Vassileva e Sun [32], mostrou-se que é possível estimular a participação e a qualidade do ensino utilizando comunidades online semelhantes aos sites de relacionamento. O comprometimento dos alunos com as comunidades das quais fazem parte incentiva a contribuição através do envio de conteúdo (mensagens, textos, vídeos, etc) com mais frequência e com maior qualidade, além de contribuir para anotar e avaliar o conteúdo enviado por outros alunos. Essa forma de aprendizagem colaborativa e ativa proporciona uma melhor assimilação do conteúdo e facilita a aquisição de habilidades cognitivas e meta-cognitivas [18]. 


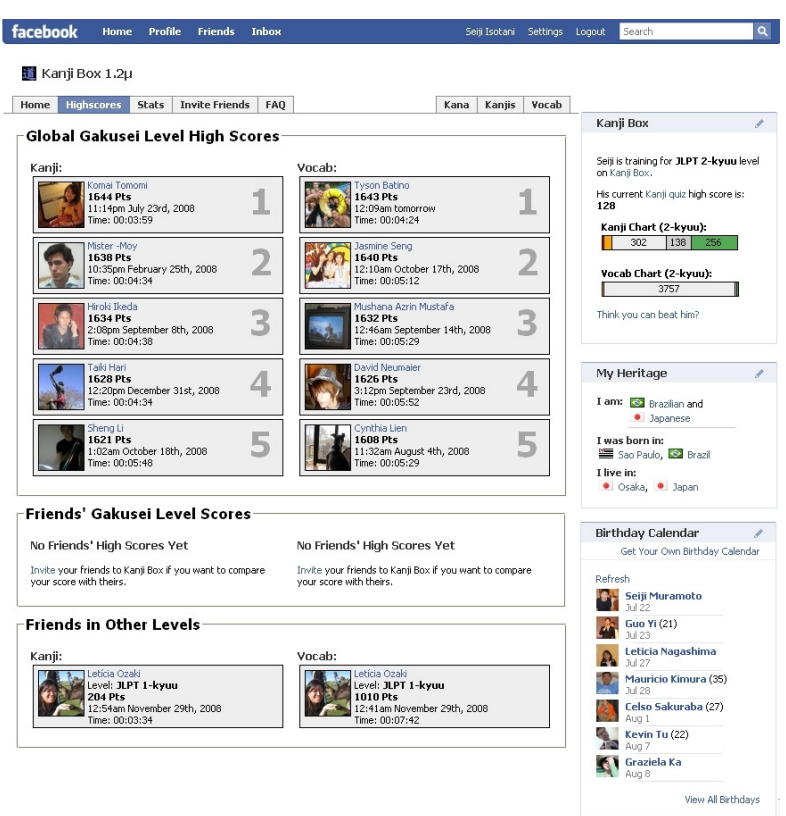

Figura 3. Kanji Box - Um programa desenvolvido para Facebook com o intuito de auxiliar o usuário a aprender Japonês.

Apesar do potencial educacional dos sites de relacionamento, existem diversos problemas relacionados ao uso indevido da informação contida neles. Além disso, o uso excessivo e, muita vezes, compulsivo; a dificuldade de controlar discussões on-line entre alunos, permitindo um comportamento não adequado; e o acesso livre à pornografia e outros conteúdos inapropriados, dificulta o uso desta ferramenta sem um controle rígido do professor, da escola e, também dos pais de alunos.

Para construir novas funcionalidades que facilitem o uso das ferramentas da Web 2.0 em ambientes de ensino é necessário uma nova arquitetura na Web na qual problemas de interoperabilidade, ambigüidade e segurança possam ser resolvidos de forma mais eficiente [20]. Além disso, para permitir que alunos e professores se beneficiem de programas mais inteligentes que semiautomaticamente consigam coletar a informação na Web e oferecer/adaptar o melhor conteúdo para se trabalhar um conceito desejado é necessário a criação de novos métodos para anotar e buscar conteúdo educacional na Web. Tais métodos devem levar em consideração diversos parâmetros como, por exemplo, aspectos culturais, estilos de aprendizagem e estratégias pedagógicas.

\section{Web 3.0 e Seu Potencial Impacto na Educação}

Embora a visão da Web Semântica e da Web Social serem antagônicas, recentemente estas duas abordagens começaram a ser utilizadas em conjunto com o intuito de promover a nova geração de tecnologias semânticas para Web [3;24]. O trabalho de Jovanovic et al. [20] aponta que através desta união é possível criar e compartilhar conteúdo de forma colaborativa e, automaticamente, representar esta informação de forma explícita e com semântica para que os computadores possam compreendê-la e utilizá-la. A Web que reune as tecnologias da Web Semântica e da Web 2.0 é conhecida como Web Semântica Social, ou Web 3.0.

Na Web Semântica Social, uma nova classe de sistemas, os chamados sistemas de conhecimento coletivo, está em fase de desenvolvimento. Estes sistemas serão capazes de auxiliar na produção de conhecimento coletivo através da análise da contribuição colaborativa humana. Segundo Gruber [17], o surgimento destes sistemas inteligentes transformará a atual inteligência acumulada encontrada na Web 2.0 em inteligência coletiva. Ou seja, a informação criada por um grupo de pessoas poderá gerar "conhecimento" que será utilizado por outras pessoas e por sistemas inteligentes para facilitar a produção de mais conhecimento. Na Figura 4, temos a visão de diversos pesquisadores sobre o rumo esperado das pesquisas na Web nos próximos 10 anos [7]. Nesta figura, o eixo $y$ representa as pesquisas em Web semântica e no eixo $x$ as pesquisas em Web 2.0. Assim, a seta na diagonal mostra o rumo das pesquisas atuais onde há uma interseção entre Web Semântica e Web Social.

Existem diversas posssiblidades de aplicação da Web 3.0 e dos sistemas de conhecimento coletivo com foco educacional. Apesar de recentes, as pesquisas nesta área já começam a oferecer soluções para diversos problemas encontrados nos sistemas educacionais atuais baseados na Web. Por exemplo, é possivel ajudar o aluno a encontrar respostas para suas dúvidas e, além disso, existe a possibilidade de proporcionar suporte pedagógico individualizado, já que o ambiente computacional tem conhecimento do problema do aluno. Outras facilidades apontadas por [12] e [14] são (a) a possibilidade de buscar semanticamente por conteúdo criado pelos alunos e professores; e (b) criar atividades nas quais os alunos possam desenvolver seu conhecimento e suas habilidades de forma ativa e colaborativa através de trabalhos em grupo com suporte computacional inteligente. Neste caso, o ambiente pode oferecer ajuda ao grupo ou a um aluno em particular ao identificar problemas na contrução ou compartilhamento da informação.

Atualmente, as pesquisas na área de Web Semântica Social com aplicação educacional têm se concentrado no desenvolvimento de blogs e Wikis semânticos. Por exemplo, nos trabalhos de Schaffert et al. [30], Ghali et al. [16] e Dimitrova et al. [14] desenvolveram-se Wikis semânticos para facilitar a aprendizagem e a construção de conhecimento em comunidades de prática (communities of 


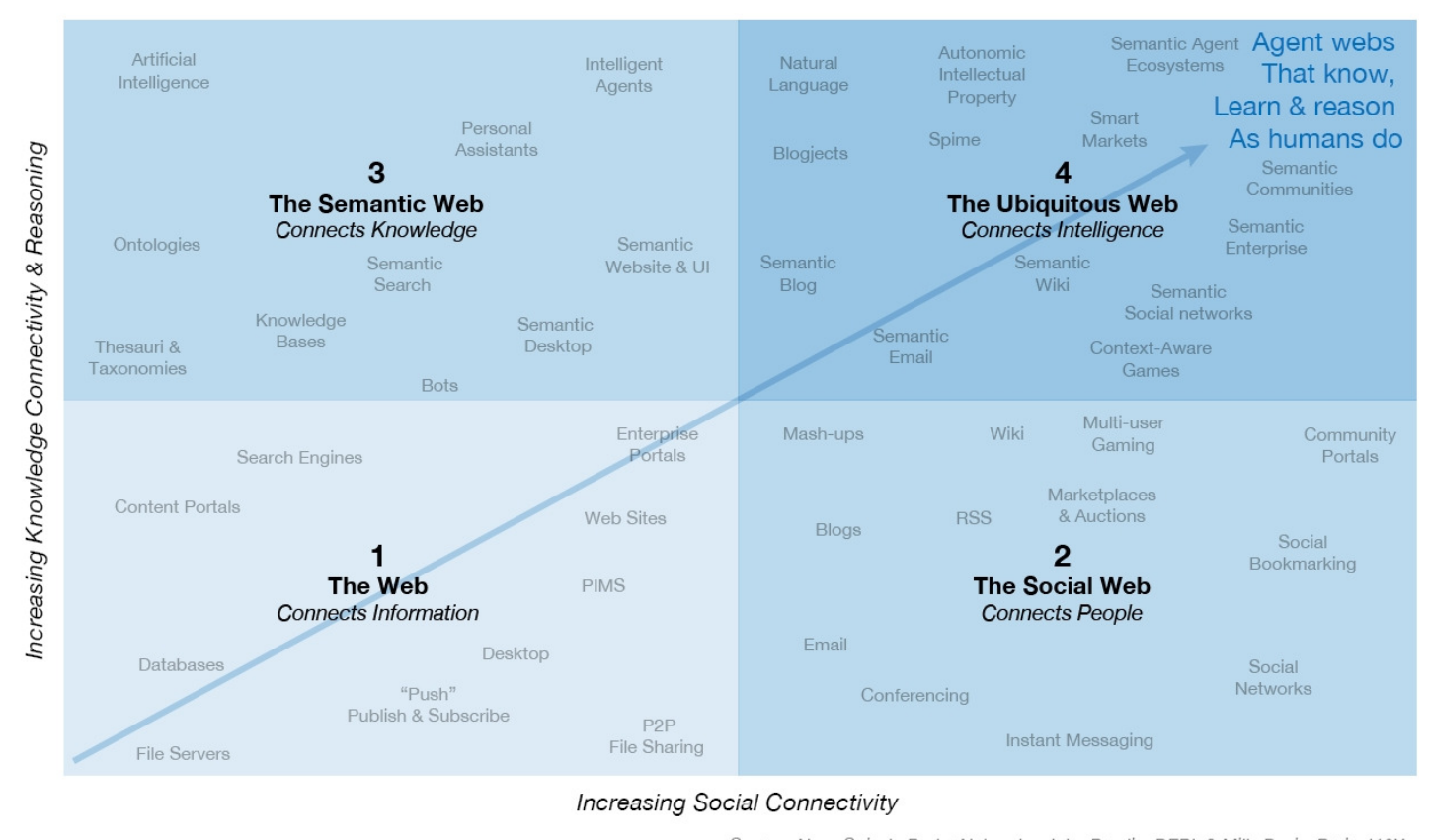

Figura 4. Visão do rumo das pesquisas na Web até 2020 de acordo com [7].

practice). Os resultados obtidos por esses trabalhos sugerem que as novas tecnologias da Web 3.0 possam transformar/melhorar a educação, porém salientam que ainda existem muitos desafios a serem superados, como: (a) o suporte ao processo de aprendizagem coletiva; (b) o uso adequado das ontologias e das anotações para melhorar as práticas de ensino-aprendizagem; e finalmente (c) o desenvolvimento e a aplicação de novos modelos pedagógicos apropriados para trabalhar com essas novas tecnologias.

Uma outra contribuição da Web 3.0 é apresentada por [30]. Neste trabalho, desenvolve-se um arcabouço que utiliza ontologias e rótulos oferecidos pelos próprios alunos para facilitar a identificação de problemas de aprendizagem e melhorar o feedback oferecido por sistemas virtuais de aprendizagem. Contudo, as pesquisas em Web Semântica Social ainda estão em fase inicial, contudo podemos identificar alguns dos principais desafios que precisam ser superados::

- Facilitar e padronizar a representação do modelo do aluno. Dessa forma, qualquer sistema poderá interagir com outro sistema para trocar informações e atualizar o modelo. Isso permitirá que se aumente a capacidade de representação e consistência do modelo viabilizando uma personalização mais eficiente que transcende as barreiras de um único sistema.
- A representação das estratégias pedagógicas em um formato que o computador possa compreender, executar e reutilizar em diferentes contextos. Para atingir este objetivo será necessário que especialistas em Educação (professores, instrutores e pedagogos) e representação de conhecimento (engenheiros e cientistas da computação) trabalhem em conjunto para formalizar uma linguagem e uma estrutura que computadores e pessoas possam utilizar e se beneficiar.

- Promover o Aprendizado em qualquer lugar, a qualquer hora, com qualquer equipamento e livre para todos. Através dos mecanimos que a Web 3.0 propõe, um aluno poderá interagir com diferentes pessoas e ambientes educacionais, utilizando diferentes dispositivos que tenham acesso a Internet (computador, celular ou qualquer outro dispositivo móvel). Além disso, a interface e o conteúdo serão automaticamente personalizados levando em consideração o modelo do aluno (que será aberto, flexível e compartilhado). O conteúdo educacional será qualquer informação contida na Web. Assim, os ambientes educacionais podem oferecer ajuda inteligente aos alunos, seguindo estratégias pedagógicas e sugerindo de forma adequada recursos Web como blogs, Wikis, vídeos, fotos, sons, e outros objetos de aprendizagem relacionados.

\section{Conclusão}

As pesquisas em Web Semântica e Web Social têm 
oferecido novas perspectivas para a educação na Web. Por um lado, a Web Semântica oferece padrões e estruturas formais para representar e anotar o conteúdo educacional de forma semântica, permitindo que um computador ou agentes de software possam "compreender" e utilizar adequadamente a informação na Web. Dessa foram, viabiliza-se a criação de sistemas educacionais mais inteligentes e com maior interoperabilidade. Por outro lado, a Web Social transformou a maneira como as pessoas interagem, trazendo diversas facilidades para criar e compartilhar conteúdo de forma interativa e colaborativa. Na Web Social, alunos e professores participam ativamente para produzir/avaliar o conteúdo e desenvolver habilidades e conhecimento de forma coletiva. A Web Semântica Social que reune estas duas linhas de pesquisa tem recebido grande atenção da comunidade internacional e seus potenciais resultados poderão modificar a forma como interagimos e aprendemos em ambientes baseados na Web.

Apesar da crescente importância no cenário internacional, existem poucas pesquisas nacionais relacionadas aos temas de Web Semântica e Web 3.0 com aplicação educacional. Dessa forma, o principal objetivo deste artigo foi apresentar o estado da arte e os desafios a serem resolvidos na área de Web Semântica, Web Social (Web 2.0) e Web Semântica Social (Web 3.0) com foco em educação e, assim, motivar e promover mais pesquisas nacionais nestas áreas em crescente expansão. A comunidade Brasileira em informática na Educação está em crescente expansão e possui o potencial para resolver os diversos problemas apresentados propondo soluções que possivelmente terão impacto muito positivo na qualidade do ensino oferecido pela Web.

\section{Referências}

[1] Berners-Lee, T., Hendler, J. \& Lassila, O. (2001) The Semantic Web. Scientific American, 284(5), 34-43.

[2] Baader, F. \& Nutt, W. (2003) The description logic hand-book: Theory, implementation and applications: Cambridge University Press, 48100.

[3] Bojãrs, U., Breslin, J. G., Peristeras, V., Tummarello, G. \& Decker, S. (2008) Interlinking the Social Web with Semantics, IEEE Intelligent Systems, 23(3), 29-40.

[4] Bittencourt, I. I., Isotani, S., Costa, E. \& Mizoguchi, R. (2008) Research Directions on Semantic Web and Education. Journal Scientia,
19(1), 59-66.

[5] Bittencourt, I. I, Costa, E., Silva, M., \& Soares, E. (2009) A Computational Model for Developing Semantic Web-based Educational Systems. Knowledge Based System. Elsevier (in Press). http://dx.doi.org/10.1016/j.knosys.2009.02.012

[6] Bryant, S. L., Forte, A., Bruckman, A. (2005) Becoming Wikipedian: Transformation of Participation in a Collaborative Online Encyclopedia. Proceedings of ACM Group Conference, 110.

[7] Davis, M. (2008) Project 10X's Semantic Wave 2008 Report. Available at http://www.project10x.com/

[8] Devedzic, V. (2006) Semantic Web and Education. Springer.

[9] Devedzic, V., Jovanovic, J., Gasevic, D., "The pragmatics of current e-learning standards," IEEE Internet Computing, Vol. 11, No. 3, 2007, pp. 16-24

[10] De Bra, P, Aroyo L., \& Chepegin, V. (2004) The Next Big Thing: Adaptive Web-Based Systems. Journal of Digital Information, 5(1). Available at http://jodi.tamu.edu/Articles/v05/i01/DeBra/

[11] Denaux, R., Dimitrova, D., \& Aroyo, L. (2005) Integrating Open User Modeling and Learning Content Management for the Semantic Web. In Proc. of Int. Conference on User Modeling, LNCS 3538, 9-18.

[12] Dichev, C., Dicheva, D. \& Fischer, J. (2007) Identity: How To Name It, How To Find It. In Workshop Proceedings of the International World Wide Web conference (WWW). Available http://ceur-ws.org/Vol-249/submission_133.pdf

[13] Dicheva, D. \& Dichev, C. (2006). TM4L: Creating and Browsing Educational Topic Maps. British Journal of Educational Technology, 37(3), 391-404.

[14] Dimitrova, V., Lau, L. \& Bek, A. L. (2008) Sharing of Community Practice through Semantics: A Case Study in Academic Writing. In Proceedings of the International Workshop on Ontologies and Semantic Web for E-Learning (SWEL), 30-39. 
[15] Eap, T., Hatala, M., Gasevic, D. (2008) Technologies for Enabling the Sharing of Leaning Objects. International Journal of Advanced Media and Communication, 2(1) 1-19.

[16] Ghali, A. E., Tifous, A., Buffa, M., Giboin, A. \& Dieng-Kuntz R. (2007) Using a Semantic Wiki in Communities of Practice. In Proc. of the Workshop on Building Technology Enhanced Learning Solutions for Communities of Practice, 22-31.

[17] Gruber, T. (2008) Collective knowledge systems: Where the Social Web meets the Semantic Web. Journal of Web Semantics 6(1), 4-13.

[18] Isotani, S. \& Mizoguchi, R. (2008) TheoryDriven Group Formation. In Proceedings of the International Conference on Intelligent Tutoring Systems, LNCS 5091, 646-655.

[19] Isotani, S. \& Brandão, L. O. (2006) Como usar a Geometria Dinâmica? O Papel do Professor e do Aluno Frente às Novas Tecnologias. Workshop de Informática na Educação. Anais do Congresso da Sociedade Brasileira de Computação, 120128.

[20] Jovanovic, J., Torniai, C., Gasevic, D., Bateman, S. \& Hatala, M. (2008) Leveraging the Social Semantic Web in Intelligent Tutoring Systems. In Proceedings of the International Conference on Intelligent Tutoring Systems, LNCS 5091, 563-572.

[21] Leuf, B. and Cunningham, W. The Wiki Way. Addison-Wesley: Boston, MA, 2001.

[22] Martin D, Paolucci, M., McIlraith, S., Burstein, M., McDermott, D., McGuinness, D., Parsia, B., Payne, T., Sabou, M., Solanki, M., Srinivasan, N. \& Sycara, K. (2004) Bringing Semantics to Web Services: The OWL-S Approach, Proceedings of the Int. Workshop on Semantic Web Services and Web Process Composition, 26-42.

[23] McIlraith, S. A., Son, T. C., Zeng, H.: Semantic Web Services. IEEE Intelligent Systems 16(2): 46-53 (2001)

[24] Mizoguchi, R., Hayashi, Y. and Bourdeau, J. (2007) Inside Theory-Aware Authoring System. In Proceedings of the Int. Workshop on Ontologies and Semantic Web for E-Learning (SWEL), $1-18$.

[24] Mikroyannidis, A. (2007) Toward a Social Semantic Web. IEEE Computer 40(11), 113-115.
[25] Motta, E., Domingue, J., Cabral, L. \& Gaspari, M. (2003) IRS-II: A framework and Infrastructure for Semantic Web Services. In Proc. of the Int. Semantic Web Conference (ISWC), LNCS 2870, 306-318.

[26] Murugesan, S. (2007) Understanding Web 2.0. IEEE IT Professional, 9( 4), 34-41.

[27] OWL (2008) Web Ontology Language. http://www.w3.org/TR/owl-features/

[28] Richardson, W. (2006) Blogs, Wikis, Podcasts and Other Powerful Tools for the Classrooms. Corwin Press.

[29] Schaffert, S., Bischof, D., Bürger, T., Gruber, A., Hilzensauer, W. \& Schaffert, S. (2006) Learning with Semantic Wikis. In SemWiki Workshop at the European Semantic Web Conference (ESWC), 109-123.

[30] Torniai, C., Jovanovic, J., Gasevic, D., Bateman, S., \& Hatala, M. (2008) Proceedings of the IEEE Int. Conference on Advanced Learning Technologies, 389-393.

[31] Ullrich, C., Libbrecht, P. (2008) Educational Services in the ActiveMath Learning Environment. The Learning Grid Handbook: Concepts, Technologies and Applications, Volume 2, The Future of Learning, Amsterdam, IOS Press.

[32] Vassileva J. \& Sun L. (2007) Using Community Visualization to Stimulate Participation in Online Communities.e-Service Journal, 6 (1), 3-40.

[33] W3C Semantic Web Activity (2008) http://www.w3.org/2001/sw/ 